\title{
RELATOS DE PESQUISAS
AVALIAÇÃO DA ARQUITETURA DA INFORMAÇÃO EM
PORTAIS DE PERIÓDICOS:
UMA DISCUSSÃO TEÓRICA
}

\author{
Carin Cunha Rocha \\ Mestranda em Ciência da Informação pela Universidade Federal do Ceará, \\ Brasil. Bibliotecária da Universidade Federal do Maranhão, Brasil. \\ E-mail: carinrocha@hotmail.com \\ Priscila Barros David \\ Doutora em Educação pela Universidade Federal do Ceará, Brasil. \\ Professora da Universidade Federal do Ceará, Brasil. \\ E-mail: priscila@virtual.ufc.br
}

\begin{abstract}
Resumo
As tecnologias digitais de informação e comunicação (TDIC) viabilizaram o surgimento dos portais de periódicos, os quais proporcionaram avanços significativos no acesso à informação científica. Neste cenário, a Arquitetura da Informação surge com o objetivo de planejar, organizar e projetar os ambientes informacionais digitais visando melhorar a recuperação da informação e a satisfação do usuário, além de contribuir com a gestão e o desenvolvimento de novos conhecimentos. O objetivo deste artigo é discutir teoricamente a avaliação da Arquitetura da Informação em portais de periódicos e propor um modelo de avaliação a partir das abordagens arquitetural, sistêmica, informacional e pervasiva discutidas por Oliveira, Vidotti e Bentes Pinto (2015). Demonstrando o caráter complexo e interdisciplinar da Arquitetura da Informação, o estudo aponta a relevância da avaliação de portais de periódicos, os propósitos da Arquitetura da Informação, bem como das abordagens apresentadas. Metodologicamente, a pesquisa baseou-se em uma revisão bibliográfica teórica sobre Arquitetura da Informação por meio dos principais autores desta temática e também por uma revisão bibliográfica empírica para resgatar o estado da arte nessa temática, com base em pesquisas sobre avaliação da Arquitetura da Informação em portais de periódicos por meio de mecanismos de busca na Biblioteca Digital de Teses e Dissertações do IBICT e no Google Acadêmico. Como resultado, apresenta-se um modelo para a avaliação da Arquitetura da Informação por meio das abordagens mencionadas. Concluise que o modelo apresentado constitui uma perspectiva mais ampla de investigação que poderá contribuir com a literatura nesta área e apoiar novas pesquisas.
\end{abstract}

Palavras-chave: Arquitetura da informação. Portais de periódicos. Avaliação. Abordagens. Usabilidade.

\section{INFORMATION ARCHITECTURE ASSESSMENT IN JOURNALS' PORTALS: A THEORETICAL APPROACH}

\begin{abstract}
Digital information and communication technologies (TDIC) enabled the emergence of journals' portals, which provided relevant advances in scientific information access. In such a scenario, Information Architecture emerges with the objective of planning, organizing and designing digital information environments in order to improve information retrieval and user satisfaction, in addition to contributing to the management and development of new knowledge. The aim of this article is to theoretically discuss the assessment of Information Architecture in journals' portals and propose an evaluation model based on the architectural, systemic, informational, and pervasive approaches discussed by Oliveira, Vidotti, and Bentes Pinto (2015). Showing Information Architecture complexity and interdisciplinarity, the study points out the relevance of journals' portals assessment, the Information Architecture purposes as well as the approaches addressed. Methodologically, the research was based on a theoretical bibliographic review on Information Architecture according to its most important scholars and also by an empirical
\end{abstract}

Perspectivas em Gestão \& Conhecimento, João Pessoa, v. 10, n. 2, p. 223-243, maio/ago. 2020. DOI: http://dx.doi.org/10.21714/2236-417X2020v10n2p223

http://periodicos.ufpb.br/ojs2/index.php/pgc. ISSN: 2236-417X. Publicação sob Licença (cc) EY-NC-ND 
bibliographic review to reach the state of the art in this theme, based on Information Architecture assessment research in journals' portals through search engines such as the IBICT Digital Thesis and Dissertation Library and the Google Scholar. As a result, a model for the Information Architecture assessment is presented according to the mentioned approaches. The results highlight that the assessment model constitutes a broader perspective of investigation that can contribute to the literature in this area and support new research.

Keywords: Information Architecture. Journals' Portals. Assessment. Approaches. Usability.

\section{INTRODUÇÃO}

A evolução tecnológica propiciou avanços significativos em todos os campos do saber. Especificamente, o advento das tecnologias digitais de informação e comunicação (TDIC) tem desempenhado, ao longo do tempo, um papel primordial na sociedade, atingindo não somente indivíduos, mas também infraestruturas, produtos e serviços, gerando mais pesquisa, ciência e tecnologia. As TDIC possibilitaram uma dinamização do conhecimento e uma maior exigência por parte dos usuários que cada vez mais procuram por serviços informatizados.

Destaca-se nesse cenário de padronização, a organização da informação em portais, a exemplo dos portais de periódicos científicos eletrônicos, que concederam melhorias no acesso à informação científica. Surge então, a Arquitetura da Informação com o objetivo de planejar, organizar e projetar os ambientes informacionais digitais contribuindo para uma melhor recuperação da informação e visando à satisfação do usuário. Para facilitar o acesso ao conteúdo, as informações devem estar organizadas de maneira que o usuário encontre a informação que ele procura, ou seja, que facilite seu acesso e a recuperação da informação.

Diante dessas considerações, este estudo se justifica pela relevância dos portais de periódicos como fontes de pesquisa e de informação e pelo fato de a Arquitetura da Informação oferecer maior visibilidade ao conteúdo científico, o que possibilita colaborar com a comunicação científica, além de contribuir com a gestão e o desenvolvimento de novos conhecimentos. Nesse contexto, o objetivo do artigo é discutir teoricamente a avaliação da Arquitetura da Informação em portais de periódicos e propor um modelo de avaliação a partir das abordagens arquitetural, sistêmica, informacional e pervasiva discutidas por Oliveira, Vidotti e Bentes Pinto (2015).

De acordo com os autores, a abordagem arquitetural demonstra a interdisciplinaridade da Arquitetura da Informação com a Arquitetura e o Design; a abordagem sistêmica age fornecendo modos de pensar que resultam de um fundamento teórico dos sistemas e de uma necessidade de atuação no campo dos sistemas de informação; já a abordagem informacional relaciona a Arquitetura da Informação com a Biblioteconomia e a Ciência da Informação. Por fim, a abordagem pervasiva, significa um novo momento para a Arquitetura da Informação no qual se observa uma relação direta com a tecnologia, por meio da Computação Ubíqua e o Design de Serviços. Percebe-se, portanto, um diálogo complexo e interdisciplinar da Arquitetura da Informação com outras áreas do conhecimento.

A metodologia do estudo baseou-se em uma revisão bibliográfica, teórica e empírica, sobre a temática da Arquitetura da Informação e um resgate do estado da arte em trabalhos empíricos sobre a avaliação da Arquitetura da Informação em portais de periódicos.

Inicialmente, são feitas considerações gerais sobre a avaliação de portais de periódicos. Em seguida, explicam-se os propósitos da Arquitetura da Informação e detalham-se as abordagens discutidas por Oliveira, Vidotti e Bentes Pinto (2015). Depois, caracteriza-se a metodologia da pesquisa: classificação, procedimentos de coleta e análise de dados. $O$ artigo

Perspectivas em Gestão \& Conhecimento, João Pessoa, v. 10, n. 2, p. 223-243, maio/ago. 2020. 
apresenta como resultado a proposta do modelo para a avaliação da Arquitetura da Informação em portais de periódicos. Finalmente, as conclusões do estudo são apresentadas.

\section{AVALIAÇÃO DE PORTAIS DE PERIÓDICOS}

Um portal é uma página específica na Internet que serve como ponto de acesso direto a outros conjuntos de serviços e informações, contendo subdivisões sobre determinado tema ou área do conhecimento. No que se refere a periódicos científicos, um portal exerceria a função de agregador e de índice, tendo como objetivo ajudar os pesquisadores a encontrarem informações desejadas sobre autores, títulos, temas etc. Um portal de periódicos tem a funcionalidade de agregar informações, aplicações e serviços relevantes aos usuários, filtrando a variedade de informação por meio de uma interface única (GARRIDO; ROGRIGUES, 2010).

De acordo com Targino e Torres (2014), os portais fazem parte da comunicação eletrônica e, portanto, se configuram como fontes formais. Logo, os periódicos científicos eletrônicos podem ser reunidos em um portal de periódicos.

Para Silveira (2016, p. 250), um portal de periódicos é

um conjunto de periódicos científicos de diferentes áreas que seguem padrões de qualidade nacionais e internacionais, de diversas áreas do conhecimento, afiliadas a uma instituição, agrupadas sistematicamente, com equipe multidisciplinar, que oferecem serviços especializados para atender às demandas de informação dos editores, autores, avaliadores e leitores, promovendo as funções da comunicação científica e os princípios do acesso aberto à informação científica das produções científicas nacionais e internacionais.

Compartilham dessa mesma visão Gulka, Lucas e Araújo (2016) ao afirmarem que um portal de periódicos é um ambiente em acesso aberto padronizado, que auxilia o gerenciamento de periódicos por meio de serviços como hospedagem, configuração e customização, suporte a editores, preservação digital, segurança e interoperabilidade de metadados, indexação em bases de dados e sustentabilidade editorial.

De acordo com Silveira (2016), os portais de periódicos possuem as seguintes funções: educativa, tecnológica, social e política. A função educativa se caracteriza por disponibilizar produtos e serviços para o desenvolvimento da competência informacional voltada para a editoração científica; a função tecnológica tem como objetivo prover melhores recursos, tendo em vista as mudanças sociais e tecnológicas vigentes; já as funções social e política garantem o acesso às informações públicas, bem como a articulação político-institucional.

No Brasil, buscando incentivar o acesso aberto, a recomendação do Instituto Brasileiro de Informação em Ciência e Tecnologia (IBICT) para a construção, publicação e gestão de periódicos eletrônicos é a utilização do software Open Journal Systems (OJS) em ambiente customizado de acordo com a identidade visual da instituição. O OJS foi desenvolvido pelo Public Knowledge Project (PKP), da University of British Columbia, no Canadá e é conceituado por Brito et al (2018) como "uma aplicação web para a gestão completa do processo editorial de publicações periódicas, ou seja, da submissão dos artigos à publicação do fascículo". Portanto, os portais de periódicos devem utilizar essa tecnologia.

Quanto à avaliação de portais de periódicos, é necessário, primeiramente, compreender o propósito da avaliação. Kraemer (2006) afirma que a palavra avaliação vem do latim a + valere, que significa atribuir valor ou mérito ao objeto em estudo. Para a autora, avaliar é atribuir um juízo de valor sobre a propriedade de um processo para a aferição da

Perspectivas em Gestão \& Conhecimento, João Pessoa, v. 10, n. 2, p. 223-243, maio/ago. 2020. 
qualidade do seu resultado. Avaliação significa, então, uma verificação e uma estimativa de valor de alguma coisa ou de algum trabalho.

Haydt (1988) compartilha dessa mesma visão e argumenta que avaliar é julgar ou fazer a apreciação de alguém ou de alguma coisa, tendo como base uma escala de valores. Envolve a interpretação de dados quantitativos e qualitativos para obter um parecer ou julgamento de valor, tendo por base padrões ou critérios.

Percebe-se, assim, que o processo de avaliar está relacionado ao ato de refletir, de planejar e de atingir objetivos. Não constitui uma tarefa simples e exige o domínio de conhecimentos e técnicas para isso.

No contexto dos ambientes digitais, existem várias formas de avaliação, como: por meio de estatísticas, heurísticas de usabilidade, perguntas diretas aos usuários, listas de verificação, dentre outras formas. Para Furquim (2003), o tema da avaliação de sites web conta com esforços de pesquisadores com formação em Ciência da Informação, preocupados com a qualidade da informação na web, como também com pesquisadores da Ciência da Computação, especificamente que possuem experiência na área da Engenharia de Software.

Para Mostafa e Terra (1998), a avaliação de páginas web tem sido praticada através de alguns sites. Mas "a literatura mais séria sobre o assunto é assinada pelos conteudistas como bibliotecários e educadores, em geral por serem eles os profissionais tradicionalmente voltados para a seleção e transferência de fontes informacionais" (MOSTAFA; TERRA, 1998, p. 55). As autoras defendem que existe tanto avaliação de sites na literatura impressa como online. E ainda argumentam que a literatura sobre avaliação de fontes eletrônicas abrange, em menor ou maior grau, cinco critérios de fontes impressas como: acuidade, autoridade, objetividade, atualização e cobertura. Como se trata de ambiente web como meio interativo, Nascimento (2000) soma a esses critérios o nível de interatividade da página.

Tendo em vista a busca por um ambiente digital organizado e que facilite a procura da informação pelo usuário, o conceito de Arquitetura da Informação vem sendo aplicado em ambientes informacionais como por exemplo, em um portal de periódicos. Para que esses portais sejam bem utilizados, não somente pela comunidade acadêmica, mas também pela comunidade em geral, é necessário que suas interfaces estejam de acordo com os componentes essenciais da Arquitetura da Informação, propostos por Rosenfeld, Morville e Arango (2015).

Segundo Vidotti e Sanches (2007, p. 4), a aplicação da Arquitetura da Informação na criação de portais ou websites conduz melhor o usuário às informações desejadas e torna o acesso mais eficaz e preciso, com um planejamento e organização da informação digital que facilita a navegação no sistema, amenizando os problemas referentes à localização e organização das informações. "É um planejamento dos fluxos de informação e das funcionalidades de um recurso para tornar este ambiente sob medida para o usuário final".

De acordo com Toub (2000), a avaliação da Arquitetura da Informação pode ser aplicada com os seguintes objetivos: comparar websites diferentes, comparar a nova versão com a versão anterior de um site e comparar opções de design para um problema de estrutura. Ou seja, a avaliação da Arquitetura da Informação traz benefícios aos usuários por melhorar a visibilidade do conteúdo no ambiente informacional. Adicionalmente, aperfeiçoa a eficiência e a satisfação do usuário, sua facilidade de uso, ampliando sua interação com o portal e reduzindo seu tempo na busca pela informação, afinal é muito mais fácil encontrar uma informação em um ambiente organizado.

Na seção seguinte, os propósitos da Arquitetura da Informação são discutidos em mais detalhes.

Perspectivas em Gestão \& Conhecimento, João Pessoa, v. 10, n. 2, p. 223-243, maio/ago. 2020. 


\section{ARQUITETURA DA INFORMAÇÃO}

Quando se fala em ambiente digital, as informações devem ser sinalizadas e organizadas de uma maneira que o usuário encontre a informação que ele procura, ou seja, que facilite a recuperação da informação. A Arquitetura da Informação tem o objetivo de planejar, organizar e projetar os ambientes informacionais digitais de tal forma que os conteúdos buscados sejam localizados com mais facilidade.

Historicamente, em meados dos anos 70, o arquiteto Richard Saul Wurman como reação a uma sociedade que cria enormes quantidades de informação sem cuidado, sem ordem ou organização criou as expressões "Arquitetura da Informação" e "Arquiteto da Informação" (AGNER, 2009). O uso do termo informação junto com arquitetura por Wurman aconteceu, pela primeira vez, em um discurso na conferência do American Institute of Architecture, em 1976 (RESMINI; ROSATI, 2011).

Apesar de o termo Arquitetura da Informação ter sido cunhado por Richard Wurmam, sua consolidação aconteceu com os autores Louis Rosenfeld e Peter Morville, após a publicação da obra Information architecture for the world wide web, em 1998, permitindo maior visibilidade à Arquitetura da Informação. A versão atual desta obra encontra-se na quarta edição abordando a Arquitetura da Informação não somente para a web, mas além. Originários da área de Biblioteconomia e Ciência da Informação, esses autores definem a Arquitetura da Informação como

1. O projeto estrutural de ambientes de informação partilhada.

2. A combinação de organização, rotulação e esquemas de navegação dentro de sites e intranets.

3. A arte e a ciência de moldar produtos e experiências de informação para apoiar usabilidade, encontrabilidade e compreensibilidade.

4. Uma disciplina emergente e uma comunidade prática, focada em trazer para o contexto digital os princípios de design e arquitetura. (ROSENFELD; MORVILLE; ARANGO, 2015, p.31, tradução nossa).

São muitos conceitos de Arquitetura da Informação existentes na literatura. Em uma análise desses conceitos, Albuquerque e Lima-Marques (2011) concluíram que os mesmos se agrupam em três categorias: a Arquitetura da Informação como design para um ambiente específico, exemplificando o conceito de Rosenfeld, Morville e Arango (2015); como organização de espaços de qualquer tipo, segundo a proposta original de Wurman (1991) e como percepção da realidade, conforme os conceitos trazidos por Dillon (2002) ao esclarecer o significado da expressão Arquitetura da Informação.

Com base nessas diferentes perspectivas conceituais, verifica-se que a Arquitetura da Informação possui conexões com outras áreas do conhecimento. Isso é confirmado por Agner (2009, p. 79) ao afirmar que "a Arquitetura da Informação pode ser vista como a união de três campos tradicionais: a Tecnologia, o Design e o Jornalismo/Redação." Compartilham do mesmo pensamento Rosmini e Rosati (2011) ao afirmarem que a Arquitetura da Informação tem suas raízes em um grande número de diferentes disciplinas: Design, Design Visual, Biblioteconomia, Ciência da Informação, Psicologia Cognitiva, Arquitetura e provavelmente alguns outros.

Assim, a Arquitetura da Informação pode ser compreendida mediante o uso do termo "guarda-chuva" na concepção de Dillon e Turnbull (2005), sob o qual coexistem várias outras autodenominações de profissionais e de pesquisadores. (Figura 1):

Perspectivas em Gestão \& Conhecimento, João Pessoa, v. 10, n. 2, p. 223-243, maio/ago. 2020. 
Figura 1 - Modelo conceitual da Arquitetura da Informação

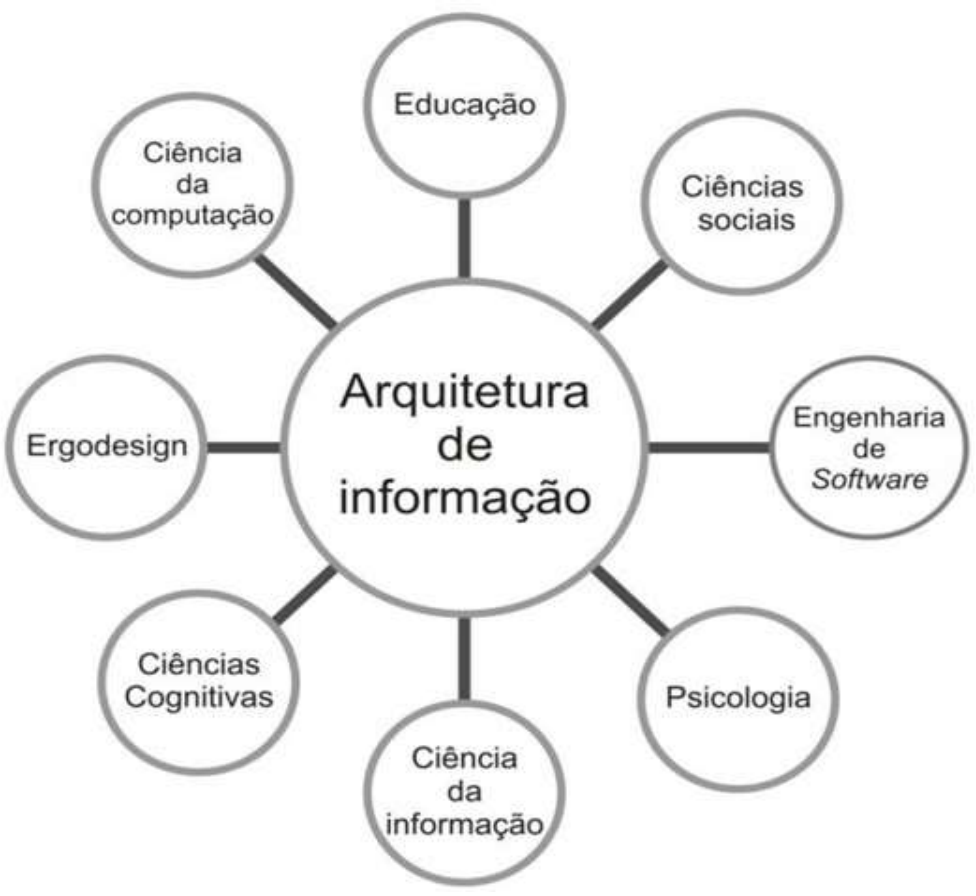

Fonte: Agner (2009, p. 77)

Diante dessas considerações, a Arquitetura da Informação aparece, portanto, como uma área interdisciplinar que utiliza vários conhecimentos de outras áreas, como a Interação Humano-Computador (IHC), a Ciência da Computação, a Comunicação e a Ciência da Informação (CAMARGO, 2010).

Nessa mesma direção, Lacerda (2015, p. 111) afirma que os métodos, modelos e teorias da Arquitetura da Informação são fortemente influenciados por ou mesmo provenientes de outras disciplinas. "Este diálogo entre disciplinas é extremamente positivo e enriquecedor, uma vez que se baseia em reciprocidade e alinhamento de fundamentos".

Portanto, as considerações desses autores permitem reconhecer que a Arquitetura da Informação compartilha e dialoga com outras áreas e disciplinas para atingir seus objetivos, configurando-a como interdisciplinar e complexa.

A respeito de interdisciplinaridade, Bentes Pinto (2007, p. 109) afirma que esta "estabelece a comunicação entre as disciplinas (no sentido científico), possibilitando o fluxo de informações entre elas, ampliando horizontes de conhecimento em uma perspectiva de seu fortalecimento". Para Nicolescu (2010), a interdisciplinaridade diz respeito à transferência de métodos de uma disciplina para outra.

Já a complexidade refere-se a uma visão interdisciplinar, com diálogo com outras áreas e disciplinas, possuindo vários aspectos ou elementos cujas relações de interdependência são incompreensíveis. Pensar a complexidade é "o maior desafio do pensamento contemporâneo que necessita de uma reforma no nosso modo de pensar" (MORIN; LE MOIGNE, 2000, p. 199). Para os autores há uma demanda das diversas áreas do conhecimento por maior correspondência entre suas disciplinas, e a interligação de suas partes concretas à totalidade. 
O pensamento complexo é, pois, essencialmente o pensamento que trata com a incerteza e que é capaz de conceber a organização. É o pensamento capaz de reunir (complexus: aquilo que é tecido conjuntamente), de contextualizar, de globalizar, mas, ao mesmo tempo, capaz de reconhecer o singular, o individual, o concreto. (MORIN; LE MOIGNE, 2000, p. 206).

Na verdade, a complexidade surge como uma espécie de confusão ou dificuldade, exprime um incômodo, uma confusão, uma incapacidade para definir de modo simples, para ordenar e clarificar as ideias. (MORIN, 2006).

Para a compreensão da complexidade, Morin (2006) destaca que é preciso entender que há um paradigma simplificador, cujo objetivo é pôr ordem no universo, expulsando dele a desordem. O princípio da simplicidade separa o que está ligado (disjunção), ou unifica o que é diverso (redução). Daí, o paradigma complexo resulta de novas concepções, de novas visões, de novas descobertas e de novas reflexões que vão se acordar, se reunir. A complexidade surge, então, para organizar as ideias para um melhor entendimento.

Na visão de Oliveira, Vidotti e Bentes Pinto (2015, p. 43)

os valores de universalidade e certeza têm dado lugar à pluralidade e à complexidade. Nessa conjuntura, a arquitetura da informação se estabelece com uma natureza inter/transdisciplinar, com seus métodos, modelos e teorias derivados do diálogo com outras disciplinas.

Nessa perspectiva, existe a contribuição de estudos para o entendimento da complexidade da Arquitetura da Informação como, por exemplo, a pesquisa de Macedo (2005, p. 15) que propôs a construção de um conceito amplo para a Arquitetura da Informação ao "estabelecer sua abrangência temática, identificar seu status científico e sintetizar os principais processos que a definem como uma prática". Ela considerou aspectos epistemológicos, científicos e práticos que contribuem para um entendimento da Arquitetura da Informação e conseguiu perceber sua relação com outras áreas do conhecimento como a Ciência da Informação, a Ergonomia e a Ciência da Computação, incluindo a subárea IHC, na qual é adotado o critério da usabilidade.

Além desse estudo, Oliveira, Vidotti e Bentes Pinto (2015) se apoiaram em uma pesquisa teórica por meio de um viés epistemológico a partir da qual traçaram as origens da Arquitetura da Informação. Os autores realizam uma contextualização espaço-temporal e a apresentam a Arquitetura da Informação sob quatro aspectos denominados abordagens: arquitetural, sistêmica, informacional e pervasiva. Tais abordagens podem ser visualizadas através do mapa conceitual representado na Figura 2: 
Figura 2 - Mapa conceitual das abordagens da Arquitetura da Informação

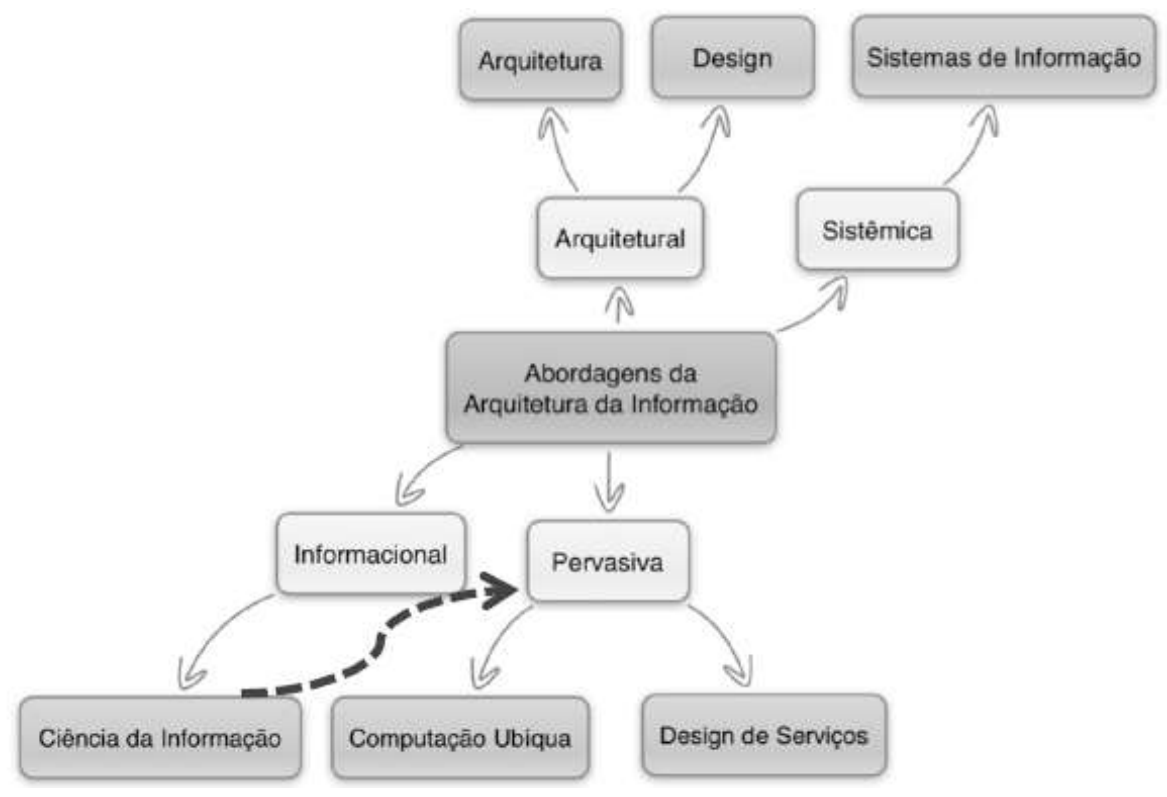

Fonte: Oliveira $(2014$, p. 83$)$

De acordo com Oliveira (2014), a compreensão da Arquitetura da Informação por meio de abordagens é uma releitura das contribuições das obras de León (2008) e de Resmini e Rosati (2011). Enquanto a abordagem arquitetural tem fundações na Arquitetura e no Design, a abordagem sistêmica tem alicerce na Teoria Geral dos Sistemas e tem ação nos sistemas de informação. Já a abordagem informacional é sedimentada na Ciência da Informação. E, por fim, a abordagem pervasiva inaugura um momento novo da Arquitetura da Informação que dialoga com a Computação Ubíqua e o Design de Serviços, sendo, portanto, uma abordagem que dialoga diretamente com a tecnologia. Cada abordagem será detalhada nas subseções seguintes.

\subsection{Abordagem arquitetural}

De acordo com Oliveira, Vidotti e Bentes Pinto (2015), esta abordagem gera uma Arquitetura da Informação com interdisciplinaridade na Arquitetura e no Design. A contribuição da Arquitetura se justifica por ser um campo devotado à racionalização dos espaços em função do uso que a sociedade ou os sujeitos the atribuem. Já o Design tem influência na Arquitetura da Informação quando se desdobra em três tendências: design de informação, de interação e gráfico. O primeiro se caracteriza pelo conteúdo informacional que será apresentado ao usuário para facilitar sua compreensão. Já o segundo torna os produtos interativos e pode ser alcançado através de duas metas: a usabilidade (a facilidade de uso) e a experiência do usuário. 0 design gráfico elabora projetos para reprodução por meios gráficos por peças extremamente comunicacionais.

Resgatando o enfoque no design de interação, representado pela usabilidade, outros autores relacionam a Arquitetura da Informação com a usabilidade. Camargo (2010) considera a usabilidade um requisito essencial para a elaboração dos elementos essenciais da 
Arquitetura da Informação, visando à facilidade de uso pelo usuário, principalmente nas questões ligadas à organização do sistema. Além disso, Macedo (2005) constatou que uma das áreas de maior relacionamento interdisciplinar com a Arquitetura da Informação é a usabilidade. Ou seja, percebe-se que a Arquitetura da Informação compartilha métodos e técnicas da usabilidade.

Para confirmar essa relação, Rosenfeld, Morville e Arango $(2015$, p.31) afirmam que a Arquitetura da Informação é "a arte e a ciência de moldar produtos e experiências de informação para apoiar usabilidade, encontrabilidade e compreensibilidade." Já Camargo e Vidotti (2011, p. 24) conceituam a Arquitetura da Informação como

\begin{abstract}
uma área do conhecimento que oferece uma base teórica para tratar aspectos informacionais, estruturais, navegacionais, funcionais e visuais de ambientes informacionais digitais por meio de um conjunto de procedimentos metodológicos a fim de auxiliar no desenvolvimento e no aumento da usabilidade de tais ambientes e de seus conteúdos.
\end{abstract}

Percebe-se nesses conceitos uma intensa relação entre a Arquitetura da Informação e a usabilidade. Isso é relevante, pois a Arquitetura da Informação objetiva organizar os ambientes informacionais digitais para melhorar a visibilidade do conteúdo visando à satisfação do usuário. A usabilidade caracteriza-se pela facilidade com que o usuário interage com o ambiente informacional digital.

A título de exemplificação desta relação, Kulpa, Pinheiro e Silva (2011) comprovam a importância das cores na usabilidade das interfaces por meio do design centrado no comportamento cultural dos usuários. Para eles, "faz parte do trabalho do designer, um bom entendimento das diferenças e demandas específicas de cada cultura, e fazê-las intrínsecas no projeto" (KULPA; PINHEIRO; SILVA, 2011, p. 133). Isso mostra que o usuário é peça chave quando se fala em Arquitetura da Informação e usabilidade.

A usabilidade pode ser avaliada por meio de técnicas, como os métodos por inspeção como a avaliação heurística proposta por Nielsen (1993), conceituada como uma técnica pela qual os avaliadores especialistas conduzem suas avaliações orientando-se por heurísticas, princípios e critérios ergonômicos propostos por especialistas na área (NIELSEN, 1993).

Observa-se que a abordagem arquitetural proposta por Oliveira, Vidotti e Bentes Pinto (2015) é pertinente, pois a Arquitetura da Informação se apoia na usabilidade, afinal uma boa apresentação visual e estrutural em um ambiente informacional digital organizado é essencial à satisfação do usuário. Na seção seguinte, será detalhada a abordagem sistêmica da Arquitetura da Informação.

\title{
3.2 Abordagem sistêmica
}

A abordagem sistêmica da Arquitetura da Informação se baseia na Teoria Geral dos Sistemas, do alemão Ludwig von Bertalanffy, que tem o objetivo de investigar a natureza dos sistemas e a interrelação entre suas partes, o que possibilita entender o objeto ou fenômeno de pesquisa. A concepção de Oliveira, Vidotti e Bentes Pinto $(2015$, p. 58) sobre esta abordagem traduz-se no fato de que "o paradigma sistêmico age na Arquitetura da Informação, fornecendo modos de pensar que resultam de uma fundamentação na teoria geral dos sistemas e de uma necessidade de atuação no campo dos sistemas de informação".

De acordo com Batista (2004, p. 13) um sistema se caracteriza pelo "conjunto de elementos interdependentes, ou um todo organizado, ou partes que interagem formando um todo unitário e complexo." Para este mesmo autor, um sistema de informação compreende 
todo e qualquer sistema que possui dados ou informações de entrada que tenham por fim gerar informações de saída para suprir determinadas necessidades.

Dessa forma, a Arquitetura da Informação trata os ambientes informacionais digitais como um conjunto de sistemas, sendo cada sistema um aglomerado de partes ou um todo organizado.

Remetendo à ideia de sistema, Rosenfeld e Morville, autores que consolidaram a Arquitetura da Informação como campo de estudos, consideraram-na uma forma de analisar os sites na internet de forma holística, ou seja, como um ambiente estruturado por partes que se inter-relacionam por meio de elementos ou componentes essenciais interdependentes, cada qual composto por regras próprias. São eles: sistema de organização, sistema de navegação, sistema de rotulação e sistema de busca.

O sistema de organização define as regras de classificação e ordenação das informações que serão apresentadas, ou seja, organizam a informação de maneira que ajude o usuário a encontrar o que precisa para atingir seu objetivo. $O$ sistema de navegação especifica a forma de se mover através do ambiente informacional digital. Podem ser de navegação principal ou embutida ou incorporada e suplementar ou remota. O sistema de rotulação define signos verbais e visuais para cada elemento informativo e de suporte à navegação do usuário, são representados pelos rótulos que podem ser textuais ou icônicos. O sistema de busca determina as perguntas que os usuários podem fazer e as respostas que irá obter nas bases de dados. Podem utilizar a linguagem natural ou os operadores booleanos (ROSENFELD; MORVILLE; ARANGO, 2015; AGNER, 2009).

A combinação desses componentes contribui com um ambiente digital planejado e organizado, que facilita as atividades de navegação e pesquisa, conduzindo o usuário a uma boa recuperação da informação, resultando na satisfação do mesmo. Na seção seguinte, serão apresentados os principais aspectos da abordagem informacional.

\title{
3.3 Abordagem informacional
}

Nessa abordagem Oliveira, Vidotti e Bentes Pinto (2015) afirmam que a Arquitetura da Informação compartilha conhecimentos da Biblioteconomia e da Ciência da Informação, compreendendo uma relação entre as áreas. E tudo se inicia pela formação acadêmica dos autores Rosenfeld e Morville, que são bibliotecários. Além disso, no Brasil, a Arquitetura da Informação se constitui atualmente como disciplina nos cursos de graduação em Biblioteconomia e nos cursos de pós-graduação em Ciência da Informação.

Oliveira, Vidotti e Bentes Pinto (2015) analisam o conceito de Arquitetura da Informação proposto por Vidotti, Cusin e Corradi (2008, p. 182)

\begin{abstract}
Arquitetura da Informação enfoca a organização de conteúdos informacionais e as formas de armazenamento e preservação (sistemas de organização), representação, descrição e classificação (sistema de rotulagem, metadados, tesauro e vocabulário controlado), recuperação (sistema de busca), objetivando a criação de um sistema de interação (sistema de navegação) no qual o usuário deve interagir facilmente (usabilidade) com autonomia no acesso e uso do conteúdo (acessibilidade) no ambiente hipermídia informacional digital.
\end{abstract}

Nesse conceito, os autores percebem vários termos que remetem à Biblioteconomia e Ciência da Informação, tais como: organização de conteúdos informacionais, preservação, representação descrição, classificação, metadados, tesauro, vocabulário controlado, recuperação, interação, acesso e uso.

Perspectivas em Gestão \& Conhecimento, João Pessoa, v. 10, n. 2, p. 223-243, maio/ago. 2020. 
Assim, torna-se pertinente citar nessa abordagem os pilares da Arquitetura da Informação (Figura 3) propostos por Rosenfeld, Morville e Arango (2015).

Figura 3 - Três pilares da Arquitetura da Informação

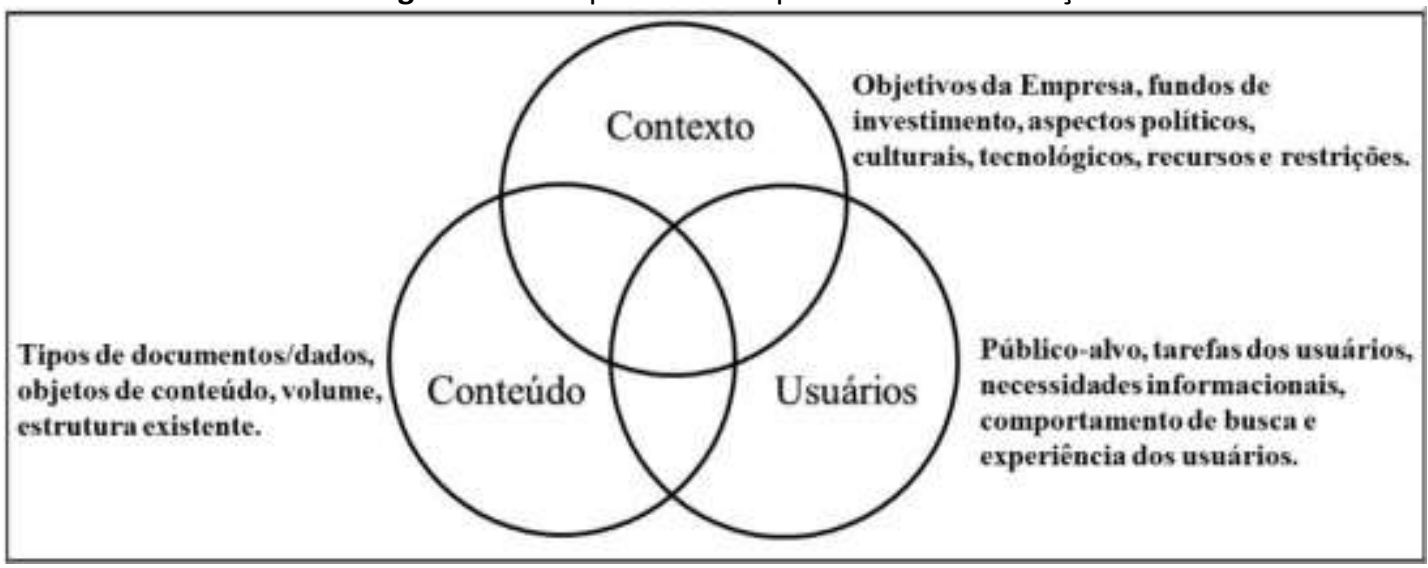

Fonte: Rosenfeld, Morville e Arango (2015, p. 39)

A Figura 3 ilustra que a Arquitetura da Informação busca compreender essas três dimensões: usuários, conteúdo e contexto. Enquanto os usuários são o público-alvo para a informação, incluindo suas necessidades, seus comportamentos e suas experiências, o conteúdo são as informações que se pretende disponibilizar. Por sua vez, o contexto compreende os objetivos do site, assim como o ambiente no qual ele se encontra.

Essa tríade forma o que Rosenfeld, Morville e Arango (2015) denominam de uma complexa e evolutiva ecologia da informação. Em suas palavras, Davenport (1998) defende uma abordagem ecológica para a Arquitetura da Informação, ao afirmar que assim como acontece com muitas abordagens orientadas para o computador, a arquitetura não chegará a lugar nenhum se não levar em consideração o comportamento e a motivação humanos. Os arquitetos precisam se comunicar com aqueles cujo comportamento será modificado continuamente, devem identificar e ouvir as pessoas que influenciarão a mudança, incluindo os usuários-alvo.

Outro aspecto relevante dessa abordagem é o fato de que os sistemas de organização, de navegação, de rotulação e de busca se apoiam nos sistemas de representação da informação como os metadados, os vocabulários controlados e os tesauros. Os metadados são constituídos como termos utilizados para descrever e representar o conteúdo dos objetos como documentos, pessoas, processos e organizações. "Metadados e vocabulários controlados apresentam uma lente fascinante através da qual podemos ver a rede de relacionamentos entre sistemas" (ROSENFELD; MORVILLE; ARANGO, 2015, p. 258).

Os metadados podem, portanto, ser aplicados no campo da Arquitetura da Informação. Conforme afirmam Rosenfeld, Morville e Arango (2015, p. 260) as "tags de metadados são usadas para descrever documentos, páginas, imagens, software, vídeo e arquivos de áudio e outros objetos de conteúdo para fins de navegação melhorada e recuperação". Em outras palavras, os metadados são estruturas para organizar a informação. Além disso, podem enfocar tanto o ponto de vista do sistema, facilitando a interoperabilidade, quanto o ponto de vista do usuário

$\mathrm{Na}$ próxima seção, serão explicados os fundamentos da abordagem pervasiva., favorecendo a obtenção de detalhes sobre a informação. 


\subsection{Abordagem pervasiva}

A abordagem pervasiva se configura como um novo momento para a Arquitetura da Informação. Oliveira, Vidotti e Bentes Pinto (2015) não a apresentam por meio de conceitos e teorias, mas sim por meio de mudanças e novos desafios que surgiram para a expansão da Arquitetura da Informação Pervasiva. Como pioneiros nessa temática, Resmini e Rosati (2011) afirmam que, a partir dos anos 2000 , houve alteração na atuação dos sujeitos com os ambientes informacionais digitais e nas experiências que os ambientes podem oferecer, sugerindo um diálogo da Arquitetura da Informação com a Computação Ubíqua e o Design de Serviços.

Essas mudanças tecnológicas que possibilitaram alterações no comportamento dos sujeitos, Resmini e Rosati (2011) denominam experiências através de canais, cross-channel. A título de exemplificação, Oliveira, Vidotti e Bentes Pinto $(2015$, p. 69) afirmam que uma experiência cross-channel pode ser vivenciada em vários ambientes, inclusive no ambiente da biblioteca. "Um usuário pode interagir com um catálogo on-line e averiguar se há títulos que Ihe interessam para posteriormente dirigir-se a ela e retirar os títulos, considerando que esse é um processo que começa digital e se torna físico".

Desse modo, as experiências cross-channel são aquelas nas quais nos movemos de um dispositivo para outro, como algo que começa digital, como um e-mail informando que um determinado produto está à venda para pegá-lo na loja física. Ou um documento sendo enviado ao e-mail após a visita a um escritório. Ou seja, são experiências que integram o físico e o digital.

Na visão de Oliveira, Vidotti e Bentes Pinto (2015),

Tais contextos tratam de experiências cross-channel, que têm sido vivenciadas atualmente e têm suscitado questionamentos sobre a Arquitetura da Informação em ambientes analógicos e digitais, no sentido de investigar formas para integrar o físico e o digital por meio do compartilhamento de camadas de informação comuns, objetivando promover experiências informacionais holísticas, ecológicas e pervasivas.

Para os autores, os trabalhos teóricos e as práticas na Arquitetura da Informação passaram a não responder mais adequadamente aos problemas tecnológicos da atualidade, sendo necessário consolidar a Arquitetura da Informação Pervasiva como uma abordagem atual que estuda as ecologias informacionais complexas, sobretudo os processos de hibridização dos lugares humanos, digitais e não digitais, em que os sujeitos vivem, trabalham e divertem-se. Isto é, uma arquitetura que seja capaz de integrar espaços, ambientes, pessoas e tecnologias de forma transparente e holística. Além disso, uma arquitetura que deve utilizar os aparatos tecnológicos de modo que se tornem invisíveis numa ecologia informacional.

Assim, a Arquitetura da Informação Pervasiva se configura como uma abordagem da Arquitetura da Informação que busca solucionar problemas tecnológicos e informacionais marcados por pervasividade. Portanto, termos computacionais como pervasividade, ubiquidade e responsividade são constantes quando se trata de Arquitetura da Informação Pervasiva. Para explicar a pervasividade, é importante destacar que a informação digital vem dominando a sociedade e a cultura, de modo que está presente nos espaços, ambiente analógicos e digitais, lugares web e não-web, em diversos tipos de dispositivos e modifica a vida dos sujeitos. Oliveira, Vidtotti e Bentes Pinto (2015) conceituam a pervasividade como a capacidade ou tendência que a informação possui de propagar-se, infiltrar-se, difundir-se total ou inteiramente através de vários meios, canais, sistemas, tecnologias etc.

Perspectivas em Gestão \& Conhecimento, João Pessoa, v. 10, n. 2, p. 223-243, maio/ago. 2020. 
Na ubiquidade, a informação se incorpora aos ambientes, ao cotidiano e ao comportamento dos sujeitos, ou seja, se integra aos diversos espaços. Refletindo sobre a ubiquidade, Lacerda e Lima-Marques (2014) afirmam que as informações estão sendo incorporadas em objetos de uso comum em toda parte. Isto muda fundamentalmente a maneira de compreender a Arquitetura da Informação, a forma de lidar com suas questões científicas e, definitivamente, a forma de praticá-la. De acordo com Oliveira, Vidotti e Bentes Pinto (2015), a ubiquidade compreende a capacidade que a informação possui de estar presente em todos os lugares ao mesmo tempo, ou seja, uma espécie de onipresença tecnológica.

E com relação à responsividade, a informação digital penetra nos mais diversos produtos tecnológicos da pós-modernidade (notebook, netbook, tablets, smarthphones, painéis digitais, televisão digital, outdoor digital, entre outros) dependendo das características do dispositivo e da capacidade de seus ambientes de informação se moldarem ao contexto e à informação. Os sujeitos poderão utilizar melhor a informação digital fazendo pontes entre esses dispositivos e seus ambientes, mas também poderão não conseguir utilizar.

Conclui-se que a pervasividade e a ubiquidade estão relacionadas à informação de forma mais ampla. Já a responsividade está ligada aos aparatos tecnológicos.

Além desses conceitos, Oliveira (2014) considerou os atributos essenciais à construção teórica sobre Arquitetura da Informação Pervasiva como, por exemplo: o termo ecologia informacional, considerada o objeto de investigação da Arquitetura da Informação Pervasiva. Caracteriza-se por um conjunto de relações intercruzadas de sujeitos, processos, estruturas informacionais, estruturas tecnológicas, espaços, ambientes, canais, dispositivos e quaisquer elementos pertencentes aos ambientes analógicos, digitais ou híbridos. Traçados por meio da noção de complexidade de forma interdependente, interativa as partes e o todo, o todo e as partes, as partes entre si. A usabilidade também é outro atributo da Arquitetura da Informação Pervasiva, pois refere-se à capacidade dos elementos da ecologia serem usados com eficiência, eficácia e satisfação pelos usuários.

Tais atributos permitiram que Oliveira (2014, p. 166, grifo nosso) conceituasse a Arquitetura da Informação Pervasiva como

uma abordagem teórico-prática da disciplina científica pós-moderna Arquitetura da Informação, trata da pesquisa científica e do projeto de ecologias informacionais complexas. Busca manter o senso de localização do usuário na ecologia e o uso de espaços, ambientes e tecnologias de forma convergente e consistente. Promove a adaptação da ecologia a usuários e aos novos contextos, sugerindo conexões no interior da ecologia e com outras ecologias. Facilita a interação com conjuntos de dados e informações ao considerar os padrões interoperáveis, a acessibilidade, a usabilidade, as qualidades semânticas e a encontrabilidade da informação, portanto deve buscar bases na Ciência da Informação.

Oliveira (2014) também elaborou um mapa conceitual da Arquitetura da Informação Pervasiva, representado na Figura 4: 
Figura 4 - Representação visual do conceito de Arquitetura da Informação Pervasiva

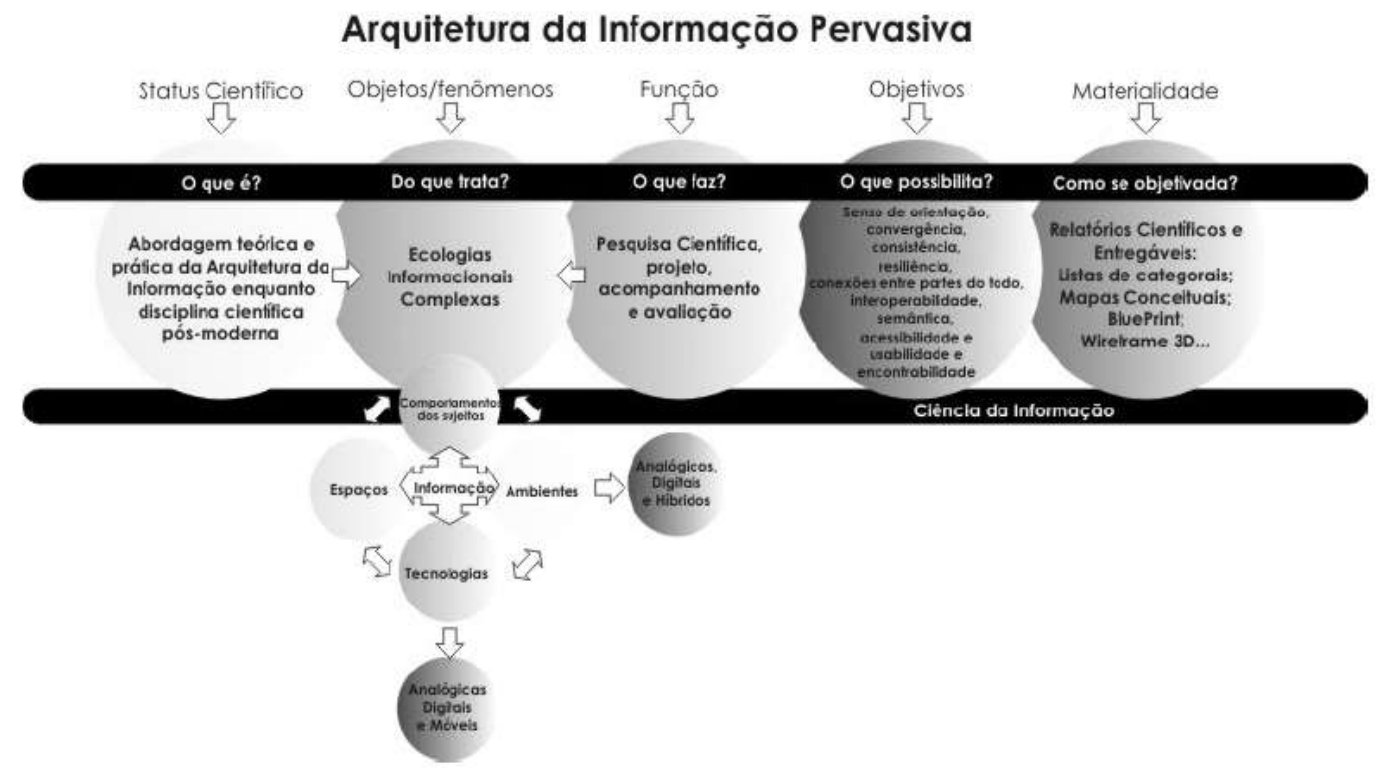

Fonte: Oliveira (2014, p. 169)

Dentro desse conceito, destaca-se o de ecologia informacional complexa. 0 termo ecologia informacional remete às contribuições de Davenport (1998) que defendia um modelo holístico de pensar e os seguintes atributos: integração dos diversos tipos de informação, reconhecimento de mudanças evolutivas, ênfase na observação, na descrição e no comportamento pessoal e informacional. Esse pensamento ilustra a Arquitetura da Informação Pervasiva como uma integração holística de espaços, ambientes, tecnologias e sujeitos com seus comportamentos através da informação. Portanto, as ecologias informacionais complexas são objeto ou fenômeno de investigação da Arquitetura da Informação Pervasiva.

Para Oliveira, Vidotti e Bentes Pinto (2015), a Arquitetura da Informação Pervasiva possibilita a realização de investigações científicas e/ou projetos de ecologias informacionais complexas que busquem: manter os sujeitos orientados dentro da ecologia; o funcionamento convergente das partes da ecologia e de seu todo em relação a outras ecologias; a adaptabilidade das partes da ecologia a novos contextos e comportamentos dos sujeitos; a interoperabilidade e a atenção às questões de semântica, usabilidade e encontrabilidade.

Percebe-se que as contribuições de Resmini e Rosati (2011), Oliveira (2014) e Oliveira, Vidotti e Bentes Pinto (2015) são pertinentes para a Arquitetura da Informação Pervasiva representando um novo momento diante das TDIC que alteraram os comportamentos dos usuários. Além disso, contribuem para novas avaliações da Arquitetura da Informação em ambientes digitais a partir dessa perspectiva.

\section{METODOLOGIA}

Este estudo parte de uma pesquisa de mestrado em desenvolvimento. Inicialmente, para estabelecer o modelo de avaliação da Arquitetura da Informação em portais de periódicos foi necessária uma pesquisa com ampla revisão bibliográfica (teórica e empírica).

A pesquisa teórica baseou-se em uma revisão de literatura sobre Arquitetura da Informação, por meio dos principais autores desta temática. Realizou-se uma busca por estudos empíricos para resgatar o estado da arte sobre a avaliação da Arquitetura da

Perspectivas em Gestão \& Conhecimento, João Pessoa, v. 10, n. 2, p. 223-243, maio/ago. 2020. 
Informação em portais de periódicos. A busca foi implementada na Biblioteca Digital de Teses e Dissertações do Instituto Brasileiro de Informação em Ciência e Tecnologia (BDTD/IBICT) e no Google Acadêmico, tomando por base o período de 2010 a 2019. Como estratégia de busca foram utilizados os seguintes termos: Arquitetura da Informação e Portais de Periódicos, separados pelo operador booleano AND.

Como resultado, foram encontrados três estudos com excelentes contribuições para a literatura científica. Entre eles, a pesquisa de Alvorcem (2010), que consistiu em avaliar o sistema de navegação no fluxo do processo editorial do Serviço Eletrônico de Editoração de Revistas (SEER) no Portal de Periódicos da Universidade Federal de Santa Catarina (UFSC) e verificar se esse sistema alcança o propósito de usabilidade junto aos editores. $\mathrm{Na}$ fundamentação teórica, a autora discutiu o SEER e abordou a Arquitetura da Informação, discutindo um de seus componentes essenciais: o sistema de navegação. Além disso, fez considerações sobre os conceitos de usabilidade e as heurísticas de Nielsen. Metodologicamente, a autora aplicou um questionário direcionado aos editores das revistas, seguindo a lógica das heurísticas de usabilidade de Nielsen, os atributos da usabilidade e os atributos do contexto de uso - NBR 9241-11; e utilizou uma ficha de avaliação (checklist), para identificar elementos da Arquitetura da Informação no sistema de navegação do SEER. Os resultados da pesquisa apontaram que o SEER alcançou o propósito de usabilidade junto aos editores. Com relação à avaliação do sistema de navegação do SEER, foram sugeridas as seguintes recomendações: revisão no sistema de navegação, rótulos mais claros, listagem de pareceristas/avaliadores, tutoriais mais explicativos, ajuda on-line, documentação e mensagens mais claras.

Alvorcem (2010) abordou o aspecto arquitetural do SEER com foco na usabilidade e enfocou o aspecto sistêmico apropriando-se apenas de um dos componentes essenciais da Arquitetura da Informação: o sistema de navegação. Ficam como lacunas do estudo a investigação das outras dimensões da Arquitetura da Informação: os aspectos informacional e pervasivo.

Outra importante contribuição para a temática da avaliação da Arquitetura da Informação em portais de periódicos foi a pesquisa de Marinho (2012), cujo objetivo consistiu em analisar a Arquitetura da Informação e a usabilidade do Portal de Periódicos da CAPES, projetando a experiência do usuário. $O$ autor discutiu elementos da Arquitetura da Informação, seus componentes essenciais, o conceito de usabilidade e seus métodos de avaliação. Como metodologia, concentrou-se em analisar o Portal de Periódicos da CAPES de acordo com os componentes essenciais da Arquitetura da Informação (sistemas de organização, de navegação, de rotulação e de busca) e realizou uma avaliação heurística de usabilidade baseada nos princípios de Nielsen, agrupados nas categorias: forma, conteúdo e comportamento. $\mathrm{O}$ resultado apontou que o Portal apresenta uma série de problemas e equívocos no que tange à Arquitetura da Informação e à usabilidade, muitos dos quais podem ser resolvidos e outros necessitam de um planejamento mais aprofundado que inclua a comunidade acadêmica. A pesquisa de Marinho (2012) pode ser considerada bastante abrangente, por ter apresentado tanto sobre o aspecto arquitetural com foco na usabilidade utilizando as heurísticas de Nielsen, quanto no aspecto sistêmico, enfocando os componentes essenciais da Arquitetura da Informação.

Nessa mesma direção, Sousa (2016) apresenta uma importante contribuição para a literatura científica da área ao propor atributos de melhores práticas para a aplicação em portais de periódicos com a finalidade de auxiliar o profissional da informação na organização e na estruturação de informações científicas. Especificamente, a autora analisou o Portal de Publicações Eletrônicas da Universidade do Estado do Rio de Janeiro (UERJ). Na fundamentação teórica abordou, dentre outros aspectos, a Arquitetura da Informação e seus componentes essenciais. Para cada componente da Arquitetura da Informação foram 
selecionados princípios e elaborados critérios específicos para a investigação do Portal de Publicações Eletrônicas da UERJ. Com base nesses critérios foi construído um instrumento de avaliação em forma de questionário, aplicado como um pré-teste a um grupo de profissionais da área de Biblioteconomia e Ciência da Informação. O resultado apontou problemas no Portal referentes: à localização (o portal de periódicos não aparece na página inicial da instituição); acessibilidade (ausência de software para atendimento aos usuários portadores de necessidades especiais); organização do conteúdo; ausência de identidade visual (falta de um logotipo) e outros. Sousa (2016) direcionou sua pesquisa para o profissional da informação abordando apenas o aspecto sistêmico.

Os estudos aqui discutidos, apesar de contribuírem com a literatura científica na área da avaliação da Arquitetura da Informação em portais de periódicos, limitaram-se a investigar o aspecto arquitetural e sistêmico, relativos à estrutura e à apresentação visual dos portais de periódicos. Para uma avaliação da Arquitetura da Informação mais ampla, é relevante a aplicação das abordagens arquitetural, sistêmica, informacional e pervasiva, conforme Oliveira, Vidotti e Bentes Pinto (2015). Esse entendimento possibilitou a elaboração da proposta de um modelo para a avaliação da Arquitetura da Informação em portais de periódicos que será explicado na seção a seguir.

\section{RESULTADO E DISCUSSÃO}

A partir do referencial teórico e metodologia discutidos nas seções anteriores deste trabalho, propõe-se um modelo para a avaliação da Arquitetura da Informação em portais de periódicos por meio das abordagens arquitetural, sistêmica, informacional e pervasiva discutidas por Oliveira, Vidotti e Bentes Pinto (2015).

É importante frisar que a compreensão da Arquitetura da Informação à luz dessas abordagens permite uma boa organização de seus conceitos e oferece um melhor entendimento acerca da avaliação de um portal de periódicos. Esse modelo, explicado no Quadro 1, mostra cada abordagem, seu objetivo e uma sugestão metodológica para investigálas:

Quadro 1 - Modelo para avaliação da Arquitetura da Informação em portais de periódicos MODELO PARA AVALIAÇÃO DA ARQUITETURA DA INFORMAÇÃO EM PORTAIS DE PERIÓDICOS

\begin{tabular}{|c|c|c|}
\hline ABORDAGEM & OBJETIVO & SUGESTÃO METODOLÓGICA \\
\hline ARQUITETURAL & $\begin{array}{l}\text { Avaliar a usabilidade do portal de } \\
\text { periódicos }\end{array}$ & $\begin{array}{c}\text { Realizar uma avaliação } \\
\text { heurística, mediante a } \\
\text { elaboração de checklist com as } \\
\text { heurísticas de Nielsen }\end{array}$ \\
\hline SISTÊMICA & $\begin{array}{c}\text { Identificar os componentes essenciais } \\
\text { da Arquitetura da Informação presentes } \\
\text { no ambiente informacional: sistema de } \\
\text { organização, navegação, rotulação e } \\
\text { busca }\end{array}$ & $\begin{array}{c}\text { Elaborar um checklist } \\
\text { contemplando os componentes } \\
\text { essenciais da Arquitetura da } \\
\text { Informação que estão presentes } \\
\text { no portal de periódicos }\end{array}$ \\
\hline INFORMACIONAL & $\begin{array}{l}\text { Caracterizar o conteúdo informacional } \\
\text { do portal de periódicos }\end{array}$ & $\begin{array}{l}\text { Mostrar ilustrações da interface } \\
\text { do ambiente, inclusive } \\
\text { apontando os componentes } \\
\text { essenciais encontrados na } \\
\text { abordagem sistêmica }\end{array}$ \\
\hline PERVASIVA & $\begin{array}{l}\text { Investigar os sujeitos, ambientes, } \\
\text { espaços e tecnologias presentes no } \\
\text { portal. }\end{array}$ & $\begin{array}{c}\text { Elaborar um mapa conceitual do } \\
\text { portal para compreendê-lo } \\
\text { como uma ecologia } \\
\text { informacional complexa }\end{array}$ \\
\hline
\end{tabular}

Fonte: Elaborado pelas autoras

Perspectivas em Gestão \& Conhecimento, João Pessoa, v. 10, n. 2, p. 223-243, maio/ago. 2020. 
Descrevendo o Quadro 1, primeiramente temos a abordagem arquitetural que relaciona a Arquitetura da Informação com a usabilidade. Pode-se, por exemplo, realizar uma avaliação heurística de usabilidade com especialistas (profissionais familiarizados com o portal de periódicos como bibliotecários, por exemplo) ou investigar a opinião dos usuários por meio de testes de usabilidade sobre sua facilidade de uso. Para a avaliação da usabilidade por especialistas, é possível elaborar um checklist contemplando as dez heurísticas de Nielsen. Os critérios que serão avaliados deverão relacionar-se a cada heurística e adicionar os graus de identificação e severidade dos problemas de usabilidade encontrados: 0 (zero), quando não há problema de usabilidade; 1 (um), quando há problema com baixa prioridade de correção; 2 (dois), quando há problema com média prioridade de correção; 3 (três), quando há problema com alta prioridade de correção. As respostas dos especialistas serão contabilizadas em porcentagens. A partir dos resultados, é possível observar os pontos fortes e os pontos frágeis do portal, os quais devem ser corrigidos, e propor melhorias.

Já a perspectiva da abordagem sistêmica contribui para a identificação dos componentes essenciais da Arquitetura da Informação presentes no portal de periódicos, como: sistema de organização, sistema de navegação, sistema de rotulação e sistema de busca, por meio de um checklist. Nesse instrumento, cada componente essencial apresenta seus elementos que serão analisados conforme a escala: atendem parcialmente, atendem plenamente, não atendem ou não são aplicados ao ambiente. Esta análise é feita pelo próprio observador, não requer a avaliação por especialistas ou usuários. A identificação permite apontar os pontos positivos do ambiente e o que pode ser melhorado visando à satisfação do usuário.

A partir da identificação destes componentes, pode-se, por meio de ilustrações da interface do portal de periódicos, apontar e sinalizar os elementos dos componentes essenciais encontrados na abordagem sistêmica. Por meio dessas ilustrações, também é possível caracterizar o conteúdo informacional do ambiente, segundo a abordagem informacional. Portanto, apenas um instrumento é capaz de atingir os objetivos tanto da abordagem sistêmica quanto da abordagem informacional.

Finalmente, os fundamentos da abordagem pervasiva permitem, por exemplo, que se compreenda o portal de periódicos como uma ecologia informacional complexa, identificando, por meio de um checklist, quais são seus sujeitos (pessoas que utilizam e fazem parte do ambiente informacional); seus espaços (quais espaços são reconhecidos no contexto do portal); seus ambientes (quais ambientes são constatados no portal) e suas tecnologias (que documentos são disponibilizados; por meio de quais dispositivos o usuário pode acessar o ambiente informacional; quais ferramentas o portal utiliza; de que forma o usuário pode ler 0 conteúdo daquele ambiente informacional). Em seguida, são organizadas e apresentadas as respostas encontradas por meio de um mapa conceitual, inspirado na pesquisa de Silva (2018), que facilita a visualização e compreensão. Tal mapa é composto por quatro cores: uma para identificar os sujeitos, outra para os ambientes encontrados, outra cor para sinalizar os espaços e uma cor que represente as tecnologias existentes.

Este modelo representa os resultados parciais de uma pesquisa de mestrado em andamento. A análise vem sendo implementada em um portal de periódicos de uma universidade pública federal, que poderá ser ampliada à investigação de outros portais de mesma natureza.

\section{CONCLUSÃO}

O propósito deste estudo foi discutir teoricamente a avaliação da Arquitetura da Informação em portais de periódicos e propor um modelo de avaliação a partir das abordagens

Perspectivas em Gestão \& Conhecimento, João Pessoa, v. 10, n. 2, p. 223-243, maio/ago. 2020. 
arquitetural, sistêmica, informacional e pervasiva discutidas por Oliveira, Vidotti e Bentes Pinto (2015).

O referencial teórico e as pesquisas empíricas que fundamentaram o estudo permitiram constatar a relevância da avaliação da Arquitetura da Informação em ambientes informacionais digitais, como os portais de periódicos e favoreceram o embasamento para a proposição de um modelo voltado a uma avaliação holística da Arquitetura da Informação nesses ambientes.

Colocar em prática a proposta de Oliveira, Vidotti e Bentes Pinto (2015) representa um avanço na literatura da área, haja vista o propósito de experimentar na prática os fundamentos dos referidos autores, além de contribuir com os portais de periódicos, suas instituições mantenedoras e seus usuários. É importante destacar o aspecto inovador do presente estudo, na medida em que apresenta uma nova forma de avaliar os ambientes informacionais digitais como um todo, contribuindo para a satisfação dos usuários.

O resultado da pesquisa evidenciou que os portais de periódicos e suas interfaces precisam estar de acordo com os requisitos propostos pela Arquitetura da Informação para que sejam bem utilizados por seus usuários.

Como limitação, esta avaliação constitui um trabalho minucioso, complexo e que demanda tempo para as análises. Não obstante, o modelo constitui uma perspectiva mais ampla de investigação, que poderá contribuir com a literatura da área e apoiar novas pesquisas. Como futuras pesquisas, pretende-se estender o modelo de investigação aqui proposto para outros espaços, tais como: portais da área da saúde, repositórios institucionais e bases de dados documentais e bibliográficas.

\section{REFERÊNCIAS}

AGNER, Luiz. Ergodesign e arquitetura da informação: trabalhando com o usuário. 2. ed. Rio de Janeiro: Quartet, 2009.

ALBUQUERQUE, Alfram Roberto Rodrigues de; LIMA_MARQUES, Mamede. Sobre os fundamentos da arquitetura da informação. Perspectivas em Gestão \& Conhecimento, João Pessoa, v. 1, Número Especial, p. 60-72, out. 2011. Disponível em: http://periodicos.ufpb.br/ojs2/index.php/pgc. Acesso em: 18 jul. 2020.

ALVORCEM, Rochelle Martins. O sistema eletrônico de editoração de revistas e sua navegabilidade: um estudo no fluxo do processo editorial no Portal de Periódicos da UFSC. 2010. 194f. Dissertação (Mestrado) - Programa de Pós-Graduação em Ciência da Informação da Universidade Federal de Santa Catarina, Florianópolis, 2010.

BATISTA, E. O. Sistemas de informação: o uso consciente da tecnologia para o gerenciamento. São Paulo: Saraiva, 2004.

BENTES PINTO, Virgínia. Interdisciplinaridade na ciência da informação: aplicabilidade sobre a representação indexal. In: PINTO, Virgínia Bentes; CAVALCANTE, Lídia Eugênia; SILVA NETO, Casemiro (orgs.). Ciência da Informação: abordagens transdisciplinares, gêneses e aplicações. Fortaleza: Edições UFC, 2007.

BRITO, Ronnie Fagundes de et al. Guia do usuário OJS 3. Brasília: IBICT, 2018. 
CAMARGO, Liriane Soares de Araújo de. Metodologias de desenvolvimento de ambientes digitais a partir dos princípios da arquitetura da informação. 2010. 287f. Tese (Doutorado em Ciência da Informação) - Universidade Estadual Paulista, Marília, 2010.

CAMARGO, Liriane Soares de Araújo de; VIDOTTI, Silvana Aparecida B. G. Arquitetura da informação: uma abordagem prática para o tratamento de conteúdo e interface em ambientes informacionais digitais. Rio de Janeiro: LTC, 2011.

DAVENPORT, Thomas H. Ecologia da informação. São Paulo: Futura, 1998.

DILLON, A. Information architecture in JASIST: Just where did we come from? Journal of the American Society for Information Science, v. 53, n. 10, p. 821, 2002.

DILLON, Andrew; TURNBULL, Don. Information Architecture. Encyclopedia of Library and Information Science, $2005 . \quad$ Disponível em: https://www.researchgate.net/publication/279439252 Information Architecture. Acesso em: 11 abr. 2019.

FURQUIM, Tatiana de Almeida. Avaliação de sites web centrada no usuário: um estudo de caso. In: ENCONTRO NACIONAL DE PESQUISA EM CIÊNCIA DA INFORMAÇÃO, 5., 2003, Belo Horizonte. Anais... Belo Horizonte: UFMG, 2003. Disponível em: http://enancib.ibict.br/index.php/enancib/venancib/paper/viewFile/2118/1253. Acesso em: 04 jun. 2019.

GARRIDO, Isadora dos Santos; RODRIGUES, Rosângela Schwarz. Portais de periódicos científicos online: organização institucional das publicações. Perspectivas em Ciência da Informação, v. 15, n. 2, p. 56-72, maio/ago. 2010.

GULKA, Juliana Aparecida; LUCAS, Eliane Rosangela Oliveira; ARAÚJO, Ronaldo Ferreira. Marketing digital em portais de periódicos científicos de acesso aberto. Cadernos de Biblioteconomia, arquivística e documentação, v. 2, p. 31-43, 2016.

HAYDT, Regina Cazaux. Avaliação do processo ensino-aprendizagem. São Paulo: Ática, 1988.

KRAEMER, Maria Elisabeth Pereira. Avaliação da aprendizagem como construção do saber. In: COLOQUIO INTERNACIONAL SOBRE GESTIÓN UNIVERSITARIA EM AMÉRICA DEL SUR, 5, 2005, Mar del Plata. Anais... Mar del Plata, 2005. Disponível em: https://repositorio.ufsc.br/handle/123456789/96974. Acesso em: 15 maio 2020.

KULPA, Cínthia Costa; PINHEIRO, Eluza Toledo; SILVA, Régio Pierre da. A influência das cores na usabilidade de interfaces através do design centrado no comportamento cultural do usuário. Perspectivas em Gestão \& Conhecimento, João Pessoa, v. 1, Número Especial, p. 119-136, out. 2011. Disponível em: http://periodicos.ufpb.br/ojs2/index.php/pgc. Acesso em: 18 jul. 2020.

LACERDA, Flávia. Arquitetura da informação pervasiva: projetos de ecossistemas na Internet das coisas. 2015. 226f. Tese (Doutorado em Ciência da Informação) - Universidade de Brasília, Brasília, 2015. 
LACERDA, Flávia; LIMA-MARQUES, Mamede. Architecture as a discipline: a methodological approach. In: RESMINI, ANDREA (org.). Reframing Information Architecture. Suíça: Springer, 2014.

LEÓN, Rodrigo Ronda. Arquitectura de Información: análisis histórico-conceptual. No sólo usabilidade Journal, n. 7, abr. 2008. Disponível em: http://www.nosolousabilidad.com/articulos/ai cc informacion.hthistoria arquitectura infor macion.htmm. Acesso em: 24 out. 2019.

MACEDO, Flávia Lacerda Oliveira de. Arquitetura da informação: aspectos epistemológicos, científicos e práticos. 2005. 190f. Dissertação (Mestrado em Ciência da Informação) Universidade de Brasília, Brasília, 2005.

MARINHO, Rafael de Barros. Arquitetura da Informação para a web: projetando a experiência do usuário no Portal de Periódicos CAPES. 2012. 142f. Dissertação (Mestrado em Ciência da Informação) - Universidade da Bahia, Salvador, 2012.

MORIN, Edgar; LE MOIGNE, Jean-Louis. A Inteligência da Complexidade. São Paulo: Petrópolis, 2000.

MORIN, Edgar. Introdução ao pensamento complexo. Porto Alegre: Sulina, 2006.

MOSTAFA, Solange P; TERRA, Marisa. As fontes eletrônicas de informação: novas formas de comunicação e de produção do conhecimento. São Paulo em perspectiva, São Paulo, v. 12, n. 4, out/dez. 1998.

NASCIMENTO, Niraldo José. Avaliação de sites sobre gestão do conhecimento na wolrd wide web: um estudo exploratório. Belo Horizonte. 2000. 230f. Dissertação (Mestrado em Ciência da Informação e Documentação) - Universidade de Brasília, Brasília, 2000.

NICOLESCU, Basarab. Methodology of transdisciplinarity-levels of reality, logic of the included middle and complexity. Transdisciplinary Journal of Engineering \& Science, v. 1, n. 1, p. 18-37, 2010.

NIELSEN, Jakob. Usability Engineering. New York: Academic Press, 1993.

OLIVEIRA, Henry P. C. de. Arquitetura da informação pervasiva: contribuições conceituais. 2014. 202f. Tese (Doutorado em Ciência da Informação) - Universidade Estadual Paulista, Marília, 2014.

OLIVEIRA, Henry P. C. de; VIDOTTI, Silvana A. B. G.; BENTES PINTO, Virgínia. Arquitetura da informação pervasiva. São Paulo: Editora UNESP; São Paulo: Cultura Acadêmica, 2015. 117 p.

RESMINI, Andrea; ROSATI, Lucas. Pervasive information architecture: designing cross-chanel user expeiences. Amsterdã: Elsevier, 2011.

ROSENFELD, Louis; MORVILLE, Peter; ARANGO, Jorge. Information architecture for the world wide web and beyond. 4 ed. Sebastopol: O'Reilly Media, 2015. 
SILVA, Mayane Paulino de Brito e. Arquitetura da informação pervasiva em repositórios digitais informacionais: o estudo de caso do Repositório da UFRN. 2018. 170f. Dissertação (Mestrado em Ciência da Informação) - Universidade Federal da Paraíba, João Pessoa, 2018.

SILVEIRA, Lúcia da. Portais de periódicos das universidades federais brasileiras: documentos de gestão. 2016. 222f. Dissertação (Mestrado em Gestão da Informação) - Universidade do Estado de Santa Catarina, Florianópolis, 2016.

SOUZA, Ester Aparecida Lima de. Aplicação da arquitetura da informação em portal de periódicos eletrônicos: o caso do Portal de Publicações Eletrônicas da Universidade Estadual do Rio de Janeiro. 2016. 164f. Dissertação (Mestrado em Ciência da Informação) Universidade Federal Fluminense, Niterói, 2016.

TARGINO, Maria das Graças; TORRES, Názia Holanda. Comunicação científica além da ciência. Revista Ação Midiática, n. 7, 2014.

TOUB, Steve. Evaluating Information Architecture: a pratical guide to assessign website organization. Argus Associates, 2000. Disponível em: https://argusacia.com/white papers/evaluating ia.pdf. Acesso em 14 maio 2020.

VIDOTTI, S. A. B. G.; SANCHES, S. A. S. Arquitetura da informação em web sites. In: SIMPÓSIO INTERNACIONAL DE BIBLIOTECAS DIGITAIS, 2004. Anais eletrônicos [...] Campinas: Unicamp, 2004. Disponível em: http://www.bibliotecadigital.unicamp.br/document/?down=8302. Acesso em: 31 out. 2019.

VIDOTTI, Silvana Aparecida Borsetti Gregório; CUSIN, César Augusto; CORRADI, Jiliane Adne Mesa. Acessibilidade digital sob o prisma da Arquitetura da Informação. In: GUIMARÃES, José Augusto Chaves; FUJITA, Mariângela Spotti Lopes. Ensino e pesquisa em Biblioteconomia no Brasil: a emergência de um novo olhar. São Paulo: Cultura Acadêmica, 2008.

WURMAN, Richard Saul. Ansiedade de Informação: como transformar informação em compreensão. São Paulo: Cultura Editora Associados, 1991. 